\title{
Spatioselective Modification of Bicompartmental Polymer Particles and Fibers via Huisgen 1,3-Dipolar Cycloaddition ${ }^{\mathrm{a}}$
}

\author{
Srijanani Bhaskar, Kyung-Ho Roh, Xuwei Jiang, Gregory L. Baker, \\ Joerg Lahann*
}

Precise nano- and microscale control of the architecture of biodegradable biomaterials is desirable for several biotechnological applications such as drug delivery, diagnostics, and medical imaging. Herein, we combine electrohydrodynamic co-jetting and highly specific surface modification (via Huisgen 1,3-dipolar cycloaddition) to prepare particles and fibers with spatioselective surface modification. We first prepared biphasic particles and fibers from commercial poly(lactide-co-glycolide) copolymers via electrohydrodynamic co-jetting of two organic solutions loaded with fluorescent macromolecules and acetylene-modified PLGA derivatives. (i) Spatially controlled reaction of poly[lactide-co-(propargyl glycolide)] with $O$-(2aminoethyl)-O'-(2-azidoethyl)heptaethylene glycol and (ii) subsequent conversion of the newly introduced amino groups with fluorescence probes resulted in particles and fibers with surface modification of one hemisphere only.

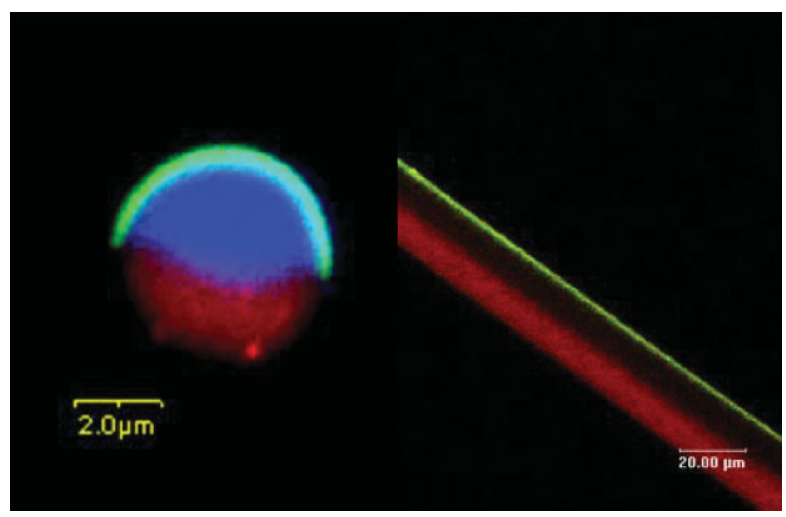

\section{Introduction}

Nano- and microstructured biomaterials with controlled chemical, physical, and biological properties have been

S. Bhaskar, K.-H. Roh, X. Jiang, J. Lahann

Departments of Macromolecular Science and Engineering, Chemical Engineering, and Materials Science and Engineering, University of Michigan, Ann Arbor, MI 48109, USA Fax: +1 (734) 764 7453; E-mail: lahann@umich.edu G. L. Baker

Department of Chemistry, Michigan State University,

East Lansing, MI-48825, USA

${ }^{a}$ Supporting information for this article is available at the bottom of the article's abstract page, which can be accessed from the journal's homepage at http://www.mrc-journal.de, or from the author. widely used for a range of different biomedical applications including tissue engineering, drug delivery, medical imaging, and diagnostics. Size, for instance, is a wellstudied parameter in the context of biomaterials development, as cellular morbidity, inflammation potential, and cellular uptake are strongly dependent on particle size. ${ }^{[1-5]}$ In addition, particle shape has been discussed as an influential materials' parameter, because phagocytosis of particles by macrophages has been shown to strongly vary with the local shape of the particle at the point where the cell is attached. ${ }^{[6]}$ Recently, however, there is growing recognition that crucial physico-chemical properties of materials may go well beyond size and shape and may include anisotropic distribution of matter, localized surface modification, or controlled compartmentalization. The increasing emphasis on anisotropic phenomena has been partially driven by the introduction of novel synthetic concepts for a range of different anisotropic 
materials. ${ }^{[7,8]}$ For instance, methods of synthesizing anisotropic particles include the use of a spinning disk, ${ }^{[9,10]}$ selfassembly, ${ }^{[11,12]}$ fusion of pre-existing particles, ${ }^{[13]}$ surface modification with partial masking, ${ }^{[14-17]}$ selective deposition, ${ }^{[18,19]}$ surface modification through partial contact with reactive media, ${ }^{[20-22]}$ microcontact printing ${ }^{[23,24]}$ and template-assisted self-assembly, ${ }^{[25,26]}$ flow-focusing lithography ${ }^{[27-29]}$ or microfluidic techniques. ${ }^{[30-33]}$ An alternative strategy towards anisotropic micro- and nanoparticles uses electrohydrodynamic co-jetting. ${ }^{[34-36]}$ In this method, the interface between two solutions is sustained during jet fragmentation and size reduction.

In principle, such novel particle geometries enable independent control of key parameters, such as chemical composition, surface functionalization, biological loading, shape, and size for each compartment, thereby effectively mimicking many of nature's complex architectures. The electrohydrodynamic co-jetting approach leads to a unique design of particles with multiple and distinct surface patterns or nano-compartments. Because of its intrinsic simplicity and generality, the electrohydrodynamic cojetting process can be applied to a wide range of specialty and non-specialty materials including many of the currently FDA approved polymer. The fact that each compartment can be designed independently from the other compartment(s) enables the combination of multiple, essential materials functions. For instance, biphasic particles that contain semiconducting polymers, hydrogels, biodegradable or bioinert polymers constitute examples that demonstrate the versatility of electrohydrodynamic cojetting. Especially, cases where incompatible materials ought to be engineered in close proximity to each other; electrohydrodynamic co-jetting can be successfully employed. Moreover, the ability to selectively modify individual areas on the surface of a particle or a fiber is another important physico-chemical property, which requires anisotropic distribution of interfacial binding sides paired with the availability of highly orthogonal immobilization chemistries.

Herein, we extend the electrohydrodynamic co-jetting process, initially established for aqueous solutions only, ${ }^{[34-36]}$ to fabrication of bicompartmental poly(lactideco-glycolide) (PLGA) fibers and particles that are prepared from organic solvents. Moreover, selective surface modification of individual compartments via surface-selective click chemistry $^{[37-42]}$ is demonstrated, yielding a novel type of spatioselectively modified particles and fibers.

\section{Experimental Part Materials}

Poly(lactide-co-glycolide) copolymers with lactide:glycolide ratios of 85:15 $\left(\bar{M}_{\mathrm{w}}=40-75 \mathrm{~kg} \cdot \mathrm{mol}^{-1}\right)$ and 50:50 $\left(M_{\mathrm{w}}=50-75 \mathrm{~kg} \cdot \mathrm{mol}^{-1}\right)$, chloroform, $N, N$-dimethylformamide (DMF), ethanol, $O$-(2-aminoethyl)-O'-(2-azidoethyl)heptaethylene glycol (azido-PEG-amine), copper sulfate $\left(\mathrm{CuSO}_{4} \cdot 5 \mathrm{H}_{2} \mathrm{O}\right)$, sodium ascorbate, fluorescein isothiocyanate (FITC), serum bovine albumin (BSA), phosphate buffered saline (PBS) and Tween-20 were purchased from SigmaAldrich, USA and used as received. Polythiophene polymers, sold under commercial names ADS 306PT $\left(\bar{M}_{\mathrm{w}}=20-70 \mathrm{~kg} \cdot \mathrm{mol}^{-1}\right)$ ADS 406PT $\left(\bar{M}_{\mathrm{w}}=30-80 \mathrm{~kg} \cdot \mathrm{mol}^{-1}\right)$, were purchased from American Dye Source, Canada. Ethyl glyoxylate (Alfa Aesar, 50 wt.-\% in toluene) was distilled prior to use.

\section{Electrohydrodynamic Jetting}

A schematic depicting the basic experimental setup for preparation of particles and fibers via electrohydrodynamic co-jetting is shown in Figure 1. Each of the two solutions was drawn in a syringe (capacity: $1 \mathrm{~mL}$, Becton-Dickinson, USA). The two syringes were held together using a syringe holder and connected to a dual cannula applicator tip (Micromedics Inc, USA) consisting of two capillaries (diameter: 26 gauge, length: $8.2 \mathrm{~cm}$ ) held together in a side by fashion via a transparent plastic tube. The needles were connected to the cathode of a DC voltage source (range: $0-30 \mathrm{kV}$, Gamma High Voltage Research, USA). The flow rate was controlled by a syringe pump (Kd Scientific, USA). The syringe pump holding the dual syringe assembly was placed atop a rectangular box-like frame with aluminium supports and Plexiglas top to facilitate control of the distance between the capillary tip and the substrate. This distance was maintained in the range of $30-35 \mathrm{~cm}$ for particles and $5-8 \mathrm{~cm}$ for fibers. A square piece of Aluminium foil was used as collecting substrate (anode). All experiments were performed at room temperature $\left(23^{\circ} \mathrm{C}\right)$ with the system placed inside a fume hood with an average face velocity of $0.1 \mathrm{~m} \cdot \mathrm{s}^{-1}$.

\section{Selective Chemical Modification of Biphasic Particles and Fibers}

Particles were harvested from the substrate with a razor blade. To $7.5 \mathrm{mg}$ of particles, azido-PEG-amine (10 times molar excess of

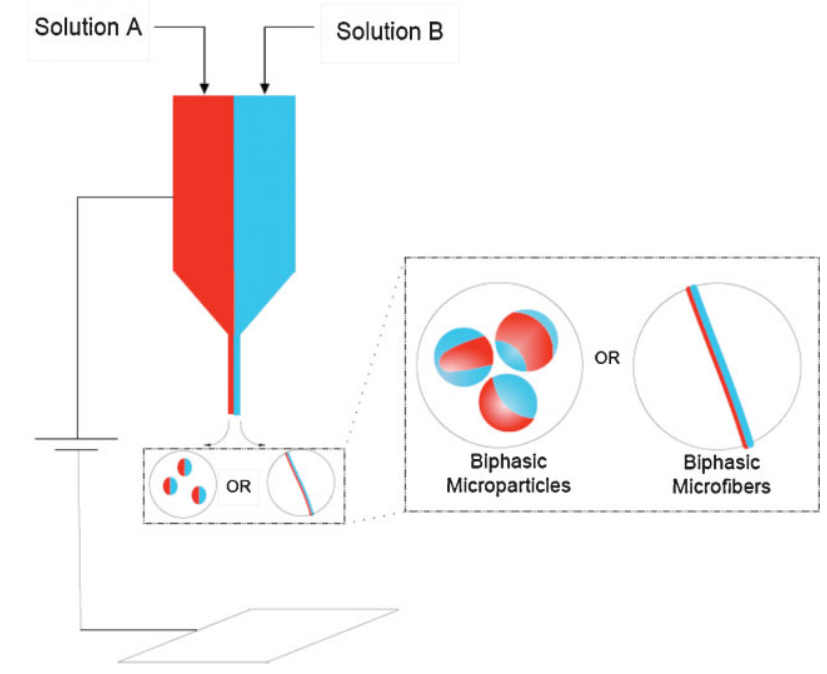

Figure 1. Scheme depicting the electrified jetting process to produce biphasic microparticles or microfibers. 
acetylene groups) was added, followed by aqueous copper sulfate solution (2 times molar excess of azide) and freshly prepared aqueous sodium ascorbate solution (100 times molar excess of $\mathrm{Cu}^{2+}$ ions). The reaction was carried out in DI water containing $0.001 \% \mathrm{v} / \mathrm{v}$ Tween-20, for $15 \mathrm{~h}$ at room temperature. The particles were purified by centrifugation with DI water, followed by repeated washing with aqueous phosphate buffer (PBS containing $0.02 \%(\mathrm{v} / \mathrm{v})$ Tween-20 and $0.1 \%(\mathrm{w} / \mathrm{v})$ BSA). Next, the purified particles were modified by addition of equimolar (w.r.t acetylene groups) amount of FITC, which was previously dissolved in ethanol. The reaction was carried out at room temperature for $5 \mathrm{~h}$, after which the particles were washed repeatedly with $2 \% \mathrm{v} / \mathrm{v}$ Tween-20 in phosphate buffered saline solution. In case of bicompartmental fibers, a fiber mesh was deposited onto the aluminium foil and was harvested by lifting the mesh off the substrate. Bicompartmental fibers were reacted and washed in 6 well plates as described for particles. The particles and fibers were then examined by confocal laser scanning microscopy as described below.

\section{Scanning Electron Microscopy}

The particles and fibers jetted on top of an aluminum substrate were sputter coated with gold and their surface morphology was examined by scanning electron microscopy (Philips XL30 ESEM, high vacuum mode).

\section{Confocal Laser Scanning Microscopy}

Samples for confocal microscopy were prepared by jetting on top of glass cover slips (24.50 mm, Fisher Scientific, USA), which, in turn, were placed on top of the aluminium substrate. The cover slips were mounted on glass slides using DI water and examined with a confocal laser scanning microscope (CLSM) (Olympus FluoView 500, USA). For the selectively modified particles, about $20 \mu \mathrm{L}$ of an aqueous particle suspension was placed onto a glass coverslip and imaged. The selectively modified fibers were detached from the foil and mounted onto glass coverslips with DI water. ADS406PT was excited by a $405 \mathrm{~nm}$ UV laser. A $488 \mathrm{~nm}$ An argon laser was used to excite ADS306PT and FITC. Optical filters for 430-460 nm, 505$525 \mathrm{~nm}$ and 560-600 nm emission wavelength were used to visualize the fluorescence of ADS 406PT, FITC and ADS306PT respectively.

\section{Results and Discussion}

Using the side-by-side configuration described in Figure 1, electrohydrodynamic co-jetting of organic solutions of mixtures of different PLGA polymers including PLGA 85:15, PLGA 50:50 and poly[lactide-co-(propargyl glycolide)] was conducted under ambient conditions. For electrohydrodynamic co-jetting, parallel extrusion of two miscible jetting solutions through a macroscopic nozzle is essential. High jetting velocities are obtained by application of electrical potential in the range of 4-6 kV between the nozzle and a counter-electrode, which acts as collection reservoir. Under these conditions, the polymer solutions are rapidly elongated resulting in a reduction in jet diameter by several orders of magnitude. Reduction in jet diameter results in rapid evaporation of the solvent and solidification of polymers and other additives to form particles or fibers with multiple compartments.

Figure $2 \mathrm{a}$ and $2 \mathrm{~b}$ show representative SEM and CLSM micrographs of biphasic microfibers and microparticles, with red and blue depicting PLGA 85:15 (labeled with ADS306PT) and PLGA 50:50 (labeled with ADS406PT) phases, respectively. Diameter and stability of the jet have a complex dependence on several solution and process parameters. The dominating solution parameters in these experiments are surface tension and conductivity, both of which depend on the solvent, which constitutes the majority of the jetting solution. PLGA microspheres have previously been made from a variety of solvents, such as chloroform, methylene chloride, ethyl acetate, acetone, and acetonitrile. ${ }^{[43]}$ We chose a mixture of 97:3 (v/v) chloroform:DMF. The use of two different solvents combines the advantages offered by a highly volatile solvent with some performance features of a less volatile solvent. Rapid evaporation of the more volatile chloroform, which constitutes the majority of the solution, increases the charge to volume ratio of the droplet, which facilitates rapid jet formation (and breakup). ${ }^{[4]}$ Instant solvent evaporation is highly desirable; in fact, this is the main factor driving the jet formation because the low conductivity $\left(\approx 0.01-0.05 \mu \mathrm{S} \cdot \mathrm{cm}^{-1}\right)$ of the organic jetting solutions limits their ability to induce charge and undergo

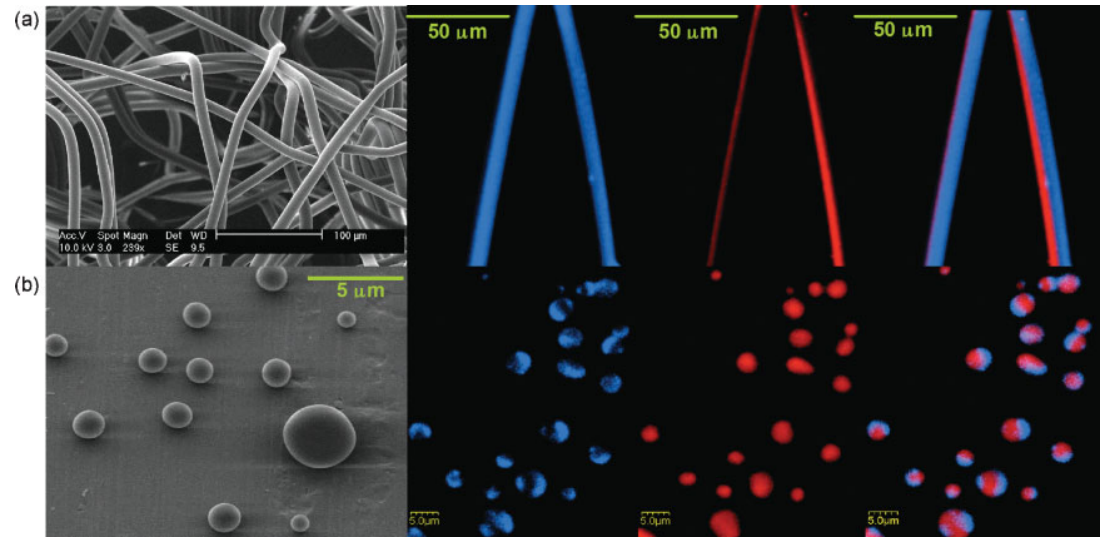

Figure 2. SEM and CLSM images of (a) biphasic microfibers, and (b) biphasic microparticles made from PLGA 85:15 (red) and PLGA 50:50 (blue). Blue fluorescence represents ADS406PT and red fluorescence represents ADS406PT. 
jet formation. On the other hand, addition of a small portion of the co-solvent, DMF increases the dielectric constant of the solution, thereby reducing jet (and particle) diameter. Being relatively non-volatile, it also enhances the long-term stability of the droplet (and hence, the Taylor cone). A 4.5\% (w/w) solution of PLGA 85:15 in one phase and PLGA 50:50 in the other, with trace amounts ( $\approx 0.01$ wt. $\%$ of PLGA) of the polythiophene polymers for imaging purposes, produced biphasic particles at a flow rate of $0.2 \mathrm{~mL} \cdot \mathrm{h}^{-1}$ (Figure $2 \mathrm{~b}$ ). As reported previously, ${ }^{[43]} \mathrm{a}$ gradual increase in the applied voltage led to the dripping mode, followed by the "cone jet mode", that is the point of formation of a Taylor cone and the appearance of a jet at its tip. Due to the lower conductivity, the base of the Taylor cone was observed to extend well into the droplet, and was not confined to the droplet tip, as it is the case of solutions with higher conductivity, such as aqueous solutions. ${ }^{[44]} \mathrm{An}$ increase in the voltage caused a lateral shift in the droplet jet, but the interface of the two solutions still remained stable, and biphasic particles could be produced. However, a further increase in voltage disrupted the interface by formation of twin cones $(>6 \mathrm{kV})$, followed by a multi-jet spray at even higher voltages $(>6.5 \mathrm{kV})$. Cone stability was enhanced by increasing the flow rates, which ensures a more sustained supply of jetting solutions. On the other hand, increasing the flow rate also resulted in an increase in jet diameters and larger average particle sizes. SEM micrographs of biphasic particles revealed spherical particles ranging from $2-5 \mu \mathrm{m}$ in diameter, with a small percentage $(<8 \%)$ of the population being comprised of flattened, donut-shaped biphasic particles of about $8 \mu \mathrm{m}$. The larger particles may be formed from an intermittent Taylor cone of partly "solidified" jetting solutions with higher apparent viscosities.

In contrast, higher polymer concentrations $(18 \% \mathrm{w} / \mathrm{w}$ PLGA in 95:5 v/v chloroform:DMF in each phase) in conjunction with lower flow rates $\left(0.02 \mathrm{~mL} \cdot \mathrm{h}^{-1}\right)$ resulted in fabrication of well-defined biphasic fibers with diameters of the order of 10-20 $\mu \mathrm{m}$ (Figure 2a). During the cojetting, a single polymer jet was ejected from the cone tip. Higher polymer concentrations resulted in greater cone stability, as compared to co-jetting of particles, and the Taylor cone was uninterruptedly maintained for several hours. In fact, bending and whipping instabilities typically associated with electrospinning ${ }^{[45,46]}$ were widely suppressed under these conditions. However, it should be pointed out that not only the lower flow rates, but also the relatively short collecting distance can contribute to the reduction of jetting instabilities. ${ }^{[46]}$ Interestingly, confocal micrographs revealed a near to perfect "half-to-half" phase distribution, which again, strongly supports the assumption that the interface between the two solutions in the droplet was maintained in a stable manner for very long periods of time.
Once the synthesis of biphasic particles and fibers based on organic solvents was established, we conducted a series of surface modification experiments to demonstrate spatioselectivity. For this purpose, we employed Huisgen 1,3-dipolar cycloaddition between azides and terminal alkynes, a widely used "click" reaction. ${ }^{[42]}$ This reaction was chosen because of its simplicity, high selectivity, and established use in a multitude of biological applications, such as targeted drug delivery or self-assembly. ${ }^{[37,40,41]}$ Prior to surface modification via click chemistry, an acetylene-modified PLGA was synthesized according to previously described protocols. ${ }^{[4]}$ Briefly, propargyl bromide was reacted with ethyl glyoxylate in the presence of activated zinc to produce 2-hydroxy-4-pentynoic acid ethyl ester. Hydrolysis of the ester in refluxing water resulted in 2-hydroxy-4-pentynoic acid, which was refluxed with catalytic $p$-toluenesulfonic acid in toluene. This cyclization reaction produced the monomer, meso/rac-3,6-di-2propynyl-1,4-dioxane-2,5-dione(propargyl glycolide). Copolymerization of propargyl glycolide with rac-lactide, catalyzed by $\mathrm{Sn}$ (2-ethylhexanoate) ${ }_{2}$ using tert-butylbenzyl alcohol as the initiator yielded poly[lactide-co-(propargyl glycolide)]. Poly[lactide-co-(propargyl glycolide)] was then added to the jetting solution of choice and was processed via electrohydrodynamic co-jetting as described for PLGA solutions.

To assess the fundamental feasibility of using the additive poly[lactide-co-(propargyl glycolide)] for modification of PLGA particles via Huisgen 1,3-dipolar cycloaddition, we first prepared monophasic particles by mixing a small amount (25\% w/w of PLGA 85:15 polymer) of poly[lactide-co-(propargyl glycolide)] in the jetting solution of PLGA 85:15. The resulting particles were then reacted with azido-PEG-amine in presence of $\mathrm{Cu}^{+}$ions (produced by reduction of $\mathrm{Cu}^{2+}$ by sodium ascorbate). The remaining free amine groups were conjugated with FITC. After surface modification, green fluorescence due to FITC was clearly seen in the confocal micrographs (Supporting Information, Figure S1a) and indicated that the particles were uniformly surface-modified. On the other hand, particles made without acetylene groups did not exhibit any green fluorescence when exposed to the same protocol (Supporting Information, Figure S1b). These initial experiments suggested that surface modification was the result of specific triazole formation rather than non-specific surface adsorption. We then applied the procedure established for monophasic particles to bicompartmental particles and fibers containing poly[lactide-co-(propargyl glycolide)] in one phase only. The amount of poly[lactideco-(propargyl glycolide)] added in one phase was $25 \%$ $(\mathrm{w} / \mathrm{w})$ with the rest being PLGA 85:15, while the second phase was loaded with 100\% PLGA 85:15. The addition of the acetylene-modified PLGA to one phase did not affect the co-jetting of either particles or fibers. Subsequent 


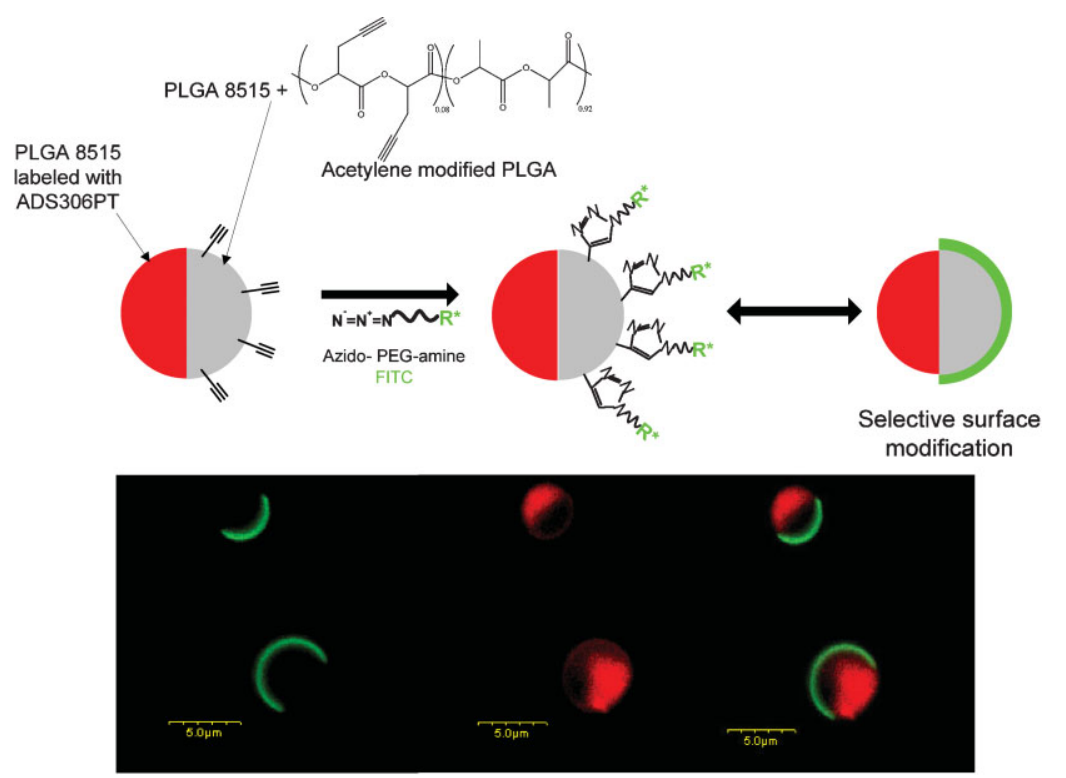

Figure 3. Biphasic microfibers labeled with ADS306PT in one phase, and containing free acetylene groups in the other phase were prepared by adding poly[lactide-co-(propargyl glycolide)] to one phase of the jetting solution. The acetylene groups were reacted with azido-PEG-amine, and the free amine groups were then reacted with FITC (PEG-amineFITC shown as $\mathbf{R}^{*}$ ). Green peripheral fluorescence on one side of the particles due to FITC indicates uniform surface modification of the acetylene containing phase.

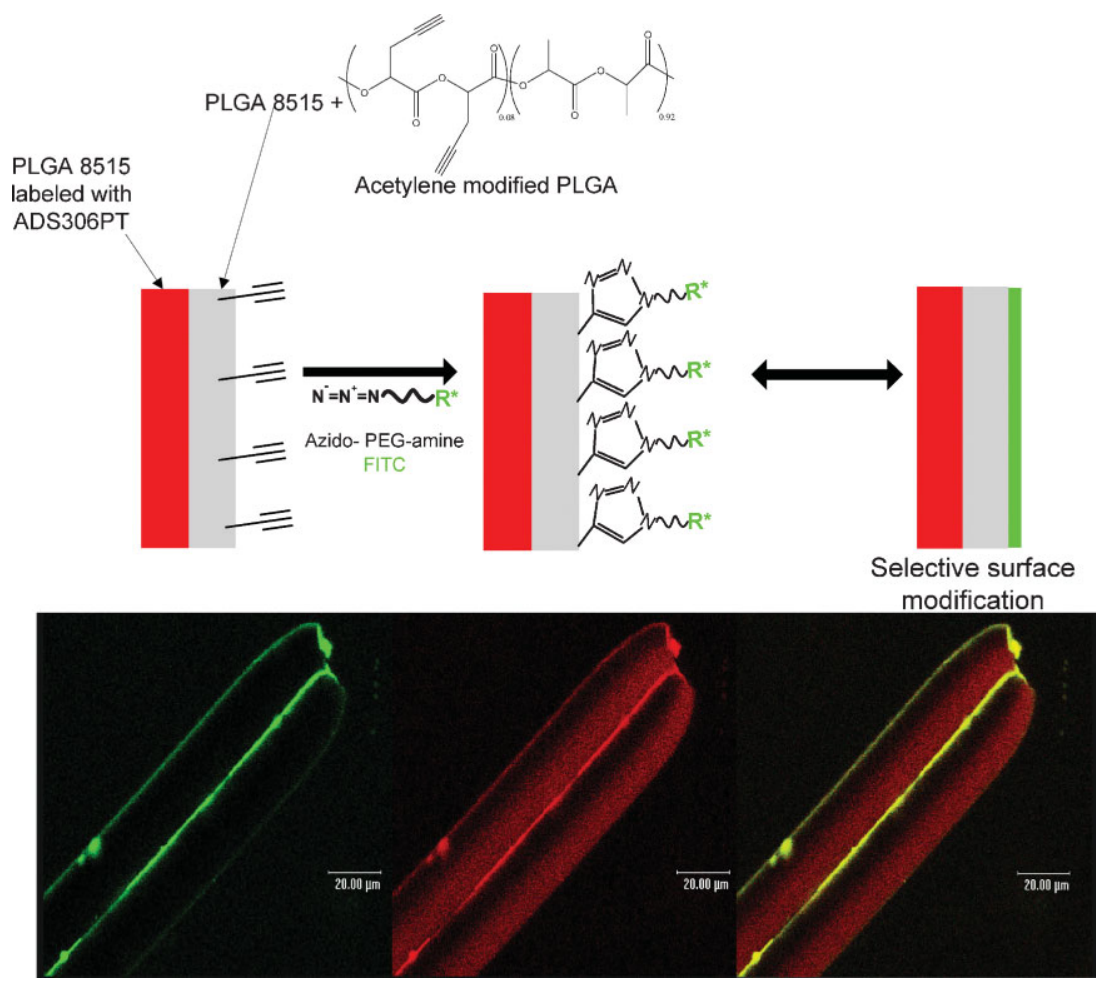

Figure 4. Selective phase modification of biphasic microfibers containing acetylene groups in one phase and ADS 306PT in the other. The acetylene containing phase was reacted with azido-PEG-amine followed by FITC (PEG-amine-FITC shown as $\mathbf{R}^{*}$ ). Green peripheral FITC fluorescence on one half of the fibers indicates spatioselective modification. analysis by confocal scanning laser microscopy revealed green fluorescence only in the compartments that contained poly[lactide-co-(propargyl glycolide)], indicating the successful modification of these compartments (Figure 3). This reaction was repeated on biphasic fibers and the same results were observed (Figure 4). Even though the acetylene-PLGA is incorporated into the bulk of the particle (or fiber), uniform fluorescence of FITC, a small molecule, suggests that a large number of acetylene groups are available on the surface for click reaction.

\section{Conclusion}

With the extension of electrohydrodynamic co-jetting for preparation of bicompartmental objects from organic solvents (in addition to previous work on aqueous biomaterials systems) ${ }^{[48]}$ and the first-time realization of bicompartmental PLGA microfibers, a new materials architecture has been realized on the basis of the well-established electrospinning technology. In the past, electrospinning has been used to prepare isotropic as well as coaxial ${ }^{[49]}$ micro- and nanofibers with potential use for various biomedical applications, such as tissue engineering. ${ }^{[50,51]}$ However, all of these scaffold materials fall short in providing spatially differentiated control of cell functions, which is believed to be a critical feature of biological scaffolds. Using electrohydrodynamic co-jetting, we have now addressed this challenge by synthesizing fibers with anisotropically distributed surface chemistries. In this work, a fluorescent ligand was selected for imaging purposes, but future bioligands may include cell adhesion peptides, antibodies, growth factors, or lectins. The herein demonstrated selective presentation of bioligands is a useful first step towards biomimetic scaffold materials, but much work still lies ahead. Beyond fiber meshes with randomly oriented fibers, well-ordered scaffolds with controlled orientation of fiber compartments relative to each other are within reach of 
this technology. The anisotropic surface modification concepts demonstrated herein on the basis of model reactions will soon be extended towards the synthesis of selectively surface modified scaffolds that can locally support promotion or inhibition of cell adhesion. Moreover, simple inclusion of environmentally responsive polymers, such as poly( $N$-isopropylacrylamide) or elastin-based polypeptides should result in biocompartmental fibers with switchable materials properties. Finally, the electrohydrodynamic co-jetting process provides a flexible platform that is not limited to the use of two jetting solutions. Extensions of this work can be easily contemplated, which will provide access to fibers with 3, 4, or even 10 compartments.

Acknowledgements: This project was funded by grants from the National Institutes of Health (5 R21 EB005732-02) and the Department of Defense (Idea Award, W81XWH-06-1-0271).

Received: July 24, 2008; Accepted: August 8, 2008; DOI: 10.1002/ marc. 200800459

Keywords: anisotropic; biodegradable; biofibers; biomaterials; click-chemistry

[1] O. Gonzalez, R. L. Smith, S. B. Goodman, J. Biomed. Mat. Res. 1996, 30, 463.

[2] I. Catelas, O. L. Huk, A. Petit, D. J. Zukor, R. Marchand, L. Yahia, J. Biomed. Mater. Res. 1998, 41, 600.

[3] V. J. Tomazic-Jezic, K. Merritt, T. H. Umbreit, J. Biomed. Mater. Res. 2001, 55, 523.

[4] A. Grandjean-Laquerriere, P. Laquerriere, M. Guenounou, D. Laurent-Maquin, T. M. Phillips, Biomaterials 2004, 26, 2361.

[5] K. Donaldson, D. Brown, A. Clouter, R. Duffin, W. MacNee, L. Renwick, L. Tran, V. Stone, J. Aerosol. Med. 2002, 15, 213.

[6] J. A. Champion, S. Mitragotri, Proc. Natl. Acad. Sci. USA 2006, 103, 4930.

[7] M. Yoshida, R. Langer, A. Lendlein, J. Lahann, Polym. Rev. 2006, 46, 347.

[8] M. Yoshida, J. Lahann, ACS Nano 2008, 2, 1101.

[9] http://www2.parc.com/hsl/projects/gyricon/, accessed Feb.2008.

[10] N. K. Sheridon, E. A. Richley, J. C. Mikkelsen, D. Tsuda, J. M. Crowley, K. A. Oraha, M. E. Howard, M. A. Rodkin, R. S. R. Swidler, Proceeding of the IDRC, SID/IEEE 1997.

[11] S. Kubowicz, J. F. Baussard, J. F. Lutz, A. F. Thunemann, H. von Berlepsch, A. Laschewsky, Angew. Chem. Int. Ed. 2005, 44, 5262.

[12] Z. Li, E. Kesselman, Y. Talmon, M. A. Hillmyer, T. P. Lodge, Science 2004, 306, 98.

[13] S.-H. Kim, G.-R. Yi, K. H. Kim, S.-M. Yang, Langmuir 2008.

[14] Z. Bao, L. Chen, M. Weldon, C. E. O. Cherniavskaya, Y. Dai, J. B. H. Tok, Chem. Mater. 2002, 14, 24.

[15] V. N. Paunov, Langmuir 2003, 19, 7970.

[16] V. N. Paunov, O. J. Cayre, Adv. Mater. 2004, 16, 788.
[17] C. Casagrande, P. Fabre, E. Raphael, M. Veyssie, Europhys. Lett. 1989, 9, 251.

[18] E. Hugonnot, A. Carles, M. H. Delville, P. Panizza, J. P. Delville, Langmuir 2003, 19, 226.

[19] H. Takei, N. Shimizu, Langmuir 1997, 13, 1865.

[20] K. Nakahama, H. Kawaguchi, K. Fujimoto, Langmuir 2000, 16, 7882.

[21] L. Petit, E. Sellier, E. Duguet, S. Ravaine, C. Mingotaud, J. Mater. Chem. 2000, 10, 253.

[22] L. Petit, J. P. Manaud, C. Mingotaud, S. Ravaine, E. Duguet, Mater. Lett. 2001, 51, 478.

[23] O. Cayre, V. N. Paunov, O. D. Velev, Chem. Commun. 2003, 2296.

[24] H. Y. Koo, D. K. Yi, S. J. Yoo, D. Y. Kim, Adv. Mater. 2004, 16, 274.

[25] L. Nagle, D. Fitzmaurice, Adv. Mater. 2003, 15, 933.

[26] Y. D. Yin, Y. Lu, Y. N. Xia, J. Am. Chem. Soc. 2001, 123, 771.

[27] A. S. Utada, E. Lorenceau, D. R. Link, P. D. Kaplan, H. A. Stone, D. A. Weitz, Science 2005, 308, 537.

[28] S. O. Xu, Z. H. Nie, M. Seo, P. Lewis, E. Kumacheva, H. A. Stone, P. Garstecki, D. B. Weibel, I. Gitlin, G. M. Whitesides, Angew. Chem. Int. Ed. 2005, 44, 724.

[29] L. Martin-Banderas, M. Flores-Mosquera, P. Riesco-Chueca, A. Rodriguez-Gil, A. Cebolla, S. Chavez, A. M. Ganan-Calvo, Small 2005, 1, 688.

[30] T. Nisisako, T. Torii, T. Higuchi, Chem. Eng. J. 2004, 101, 23.

[31] T. Nisisako, T. Torii, T. Takahashi, Y. Takizawa, Adv. Mater. 2006, 18, 1152.

[32] Z. H. Nie, W. Li, M. Seo, S. Q. Xu, E. Kumacheva, J. Am. Chem. Soc. 2006, 128, 9408.

[33] R. F. Shepherd, J. C. Conrad, S. K. Rhodes, D. R. Link, M. Marquez, D. A. Weitz, J. A. Lewis, Langmuir 2006, 22, 8618.

[34] K.-H. Roh, D. C. Martin, J. Lahann, Nat. Mater. 2005, 4, 759.

[35] K. H. Roh, D. C. Martin, J. Lahann, J. Am. Chem. Soc. 2006, 128, 6796.

[36] K. H. Roh, M. Yoshida, J. Lahann, Langmuir 2007, 23, 5683.

[37] W. H. Binder, R. Sachsenhofer, Macromol. Rapid Commun. 2008, 29, 952-981.

[38] H. C. Kolb, M. G. Finn, K. B. Sharpless, Angew. Chem. Int. Ed. 2001, 40, 2004

[39] V. V. Rostovtsev, L. G. Green, V. V. Fokin, K. B. Sharpless, Angew. Chem. Int. Ed. 2002, 41, 2596.

[40] J.-F. Lutz, Angew. Chem. Int. Ed. 2007, 46, 1018.

[41] H. Nandivada, X. Jiang, J. Lahann, Adv. Mater. 2007, 19, 2197.

[42] R. Huisgen, "1,3-Dipolar Cycloaddition Chemistry", Wiley, New York 1984, p. 1.

[43] C. Berkland, D. W. Pack, K. Kim, Biomaterials 2004, 25, 5649.

[44] M. Cloupeau, B. Prunet-Foch, J. Electrostat. 1989, 22, 135.

[45] D. H. Reneker, A. L. Yarin, H. Fong, S. Koombhongse, J. Appl Phys 2000, 87, 4531.

[46] T. Han, D. H. Reneker, A. L. Yarin, Polymer 2007, 48, 6064.

[47] X. Jiang, E. B. Vogel, M. R. Smith, III, G. L. Baker, Macromolecules 2008, 41, 1937.

[48] M. Yoshida, K. H. Roh, J. Lahann, Biomaterials 2007, 28, 2446.

[49] D. H. Reneker, I. Chun, Nanotechnology 1996, 7, 216.

[50] L. Huang, R. A. McMillan, R. P. Apkarian, B. Pourdeyhimi, V. P. Conticello, E. L. Chaikof, Macromolecules 2000, 33, 2989.

[51] E. H. Sanders, R. Kloefkorn, G. L. Bowlin, D. G. Simpson, G. E. Wnek, Macromolecules 2003, 36, 3803. 